\title{
Frequency of peri-ictal apnea and cardiac arrhythmias in epileptic seizures
}

\author{
Rady Yousif*, M. Ossama Abdulghani, A. Gaber, Naglaa El Khayat, Y. A. Abo Elnaga and \\ Mona Mokhtar Wahid El Din
}

\begin{abstract}
Background: Available data from witnessed and monitored sudden unexpected death in epilepsy (SUDEP) cases postulate that ictal central apnea (ICA) and ictal arrhythmias are the main causes of SUDEP. ICA is a frequent semiological feature of focal epilepsy and occasionally the only clinical manifestation of focal seizures. The aim of this study was to assess the frequency of ICA and cardiac arrhythmias in epileptic patients and to study the risk factors and predictors of their occurrence.
\end{abstract}

Methods: Fifty patients diagnosed with epilepsy were recruited in this study. All participants underwent prolonged surface video electroencephalography (VEEG) study using the 10-20 international electrode system with concomitant polysomnography including electrocardiography (ECG), heart rate monitoring, and peripheral capillary oxygen saturation $\left(\mathrm{SpO}_{2}\right)$ using pulse oximetry. Also inductance plethysmography was used to record chest and abdominal excursions.

Results: Complete datasets were available in 50 patients and 112 seizures were recorded. ICA occurred exclusively in focal epilepsy $(P<0.001)$. Temporal lobe epilepsy was associated with higher occurrence of ICA in comparison to extratemporal epilepsy $(P<0.001)$. In addition, seizures lateralized to the left hemisphere were associated with higher occurrence of ICA $(P<0.001)$. On the other side, tachycardia was found to be more associated with temporal lobe epilepsy and left hemispheric seizure onset $(P<0.001)$.

Conclusion: ICA occurred exclusively in focal seizures and tachycardia magnitude was more with focal seizures, and both had higher percentage in temporal lobe epilepsy in comparison to other types and in seizures with left hemispheric onset.

Keywords: Epilepsy, Central apnea, Arrhythmia, SUDEP

\section{Introduction}

Sudden unexpected death in epilepsy (SUDEP), a devastating event, is reported as the most common cause of death in epilepsy patients. Nocturnal seizures are reported as an evident risk factor for SUDEP [1]. Patients who die due to SUDEP are mostly of young age with uncontrolled epilepsy and they die particularly during convulsive seizures $[2,3]$. Cardiac and respiratory mechanisms were postulated to contribute to SUDEP pathophysiology [2, 4]. ICA

\footnotetext{
* Correspondence: radyyousif@gmail.com

Neurology Department, Faculty of Medicine, Ain Shams University, Cairo, Egypt
}

is a frequent semiological feature of focal epilepsy and was focal seizures [5]. Peri-ictal breathing dysfunction was reseizures ca potent to a fecting the autonomic nervous system. Changes in cardiac signals are reported as potential biomarkers that may resemble an extracerebral indicator of ictal onset in some patients [7]. Cardiac changes are frequently seen during ctal activity in adults, and the most frequent cardiac hange is tachycardia [8].

The aim of this study was to investigate the frequency of ICA and cardiac arrhythmias in epileptic patients 
during seizures also to study the risk factors and predictors of ICA and cardiac arrhythmias in epileptic seizures.

\section{Methods}

This study consecutively recruited 50 participants diagnosed with Epilepsy from Epilepsy outpatient clinics at Neurology Department of Ain Shams University Hospitals in the city of Cairo, from August 2018 through July 2020.

This was an observational cross-sectional study with a convenient sample. Inclusion criteria of our sample were patients with the age ranging from 10 to 40 years and age of onset since birth till 40 years. Written informed consent was obtained from all participants or the first of kin in participants less than 18 years old. Exclusion criteria were patients with movement artifacts or electrical artifacts obscuring plethysmographic signal or unavailable video, patients diagnosed with obstructive sleep apnea disorder and patients who did not develop seizure during time of recording. Also psychogenic nonepileptic seizures (PNES) were excluded.

All patients were evaluated by detailed clinical history including personal data, past history, history of current illness, status epilepticus occurrence, family history of epilepsy, and antiseizure medications used. Response to first antiseizure medication was assessed and patients were classified to be responsive or non-responsive to first antiseizure medication; responsiveness was defined as being seizure-free for a minimum of three times the longest preintervention interseizure interval or 12 months, whichever is longer in a patient receiving an antiseizure drug [9]. Also having drug-resistant epilepsy (DRE) or not was assessed, DRE was defined as failure of adequate trials of two tolerated, appropriately chosen and used antiseizure drug schedules (whether as monotherapy or in combination) to achieve sustained seizure freedom [9]. The National Hospital Seizure Severity Scale was used to assess seizure severity [10]. Prolonged nocturnal VEEG study using the10-20 international electrode system and concomitant polysomnography (using Natus, Nicolet EEG n32, USA) were done for all the participants. Duration of recording was at least 6 up to $12 \mathrm{~h}$ and at least two seizures were recorded to be included in the study. Seizures were classified as stated by the International League Against Epilepsy (ILAE) 2017 seizure classification [11]. The putative epileptogenic zone was detected in each seizure based on the concordance of seizure semiology, ictal onset recorded by EEG, and location of the lesion in magnetic resonance imaging (MRI). Concomitant polysomnography included ECG monitoring and peripheral capillary oxygen saturation $\left(\mathrm{SpO}_{2}\right)$ using pulse oximetry. Also inductance plethysmography was used to record chest and abdominal excursions. Central apnea was defined as cessation of respiratory movements lasting for $\geq 10 \mathrm{~s}$ and oxygen desaturations was classified into three levels, mild $\left(\mathrm{SpO}_{2}\right.$ of 90-94\%), moderate (75-89\%), and severe (<75\%) [6]. Cardiac arrhythmias including tachycardia and bradycardia were recorded. Tachycardia was defined as heart rate $>100$ beats per minute and bradycardia $<60$ beats per minute, or $>20 \%$ deviation from the baseline heart rate. Neuroimaging was done including MRI brain epilepsy protocol (diffusion weighted, flair axial and angled coronal, T1 axial and coronal, T2 angled coronal and T2* images) using a 1.5-T MR scanner (Achieva and Ingenia, Philips medical system, Eindhoven, Netherlands).

\section{Statistical analysis}

The collected data was revised, coded, and introduced to a PC using Statistical package for Social Science (SPSS 25 , by IBM, Armonk, NY, USA). Data were introduced and suitable analysis was done according to the type of data obtained for each parameter: mean, standard deviation $( \pm \mathrm{SD})$, and range for parametric numerical data; median and interquartile range (IQR) for non-parametric numerical data; and frequency and percentage for nonnumerical data. Chi-square test was used to assess the relationship between two qualitative variables. Student's $T$ test was used to investigate the statistical significance of the difference between two study group means. ANOVA test was used to investigate the statistical significance of the difference between more than two study group means. Correlation analysis (using Spearman's rho and Pearson method) was used to assess the strength of association between two quantitative variables. $P$ value level of significance defined $P>0.05$ as non-significant, $P<0.05$ as significant, and $P<0.01$ as highly significant.

\section{Results}

Fifty patients were included in the study, 30 males and 20 females with a mean age of $23.14 \pm 8.127$. The mean age of onset was $13.902(0.08-40)$ years. The mean duration of illness was $9.242(0.08-34)$ years. The mean frequency of seizures per month was 33.68 (2-180). Past history of status epilepticus was evident in $14(28 \%)$ patients. As regards response to first antiseizure medication, 18 (36\%) patients were responsive, 31 (62\%) patients were non-responsive, and one patient was recently diagnosed and was not on medications. Drug resistance was evident in $26(52 \%)$ patients. As regards antiseizure medications, $11(22 \%)$ patients were on single medication and 39 (78\%) patients were on multiple medication. As regards MRI brain findings, 28 (56\%) patients had normal MRI brain, 6 (12\%) patients had cortical atrophy, $5(10 \%)$ patients had mesial temporal sclerosis, 4 (8\%) patients had encephalomalacia, 4 (8\%) patients had demyelinating lesion, 2 (4\%) patients had 
cortical dysplasia, and one (4\%) patient had arteriovenous malformation (AVM).

During tracing, 112 seizures were recorded, 23 (20.54\%) seizures were focal onset with impaired awareness (FOIA) motor onset with automatism, 8 (7.14\%) seizures were FOIA non-motor onset, 20 (17.86\%) seizures were FOIA motor onset hyperkinetic or focal onset aware (FOA) motor onset tonic or clonic, 18 (16.07\%) seizures were focal onset to bilateral tonicclonic seizures (FBTCS), 19 (16.96\%) seizures were generalized onset non-motor typical seizures (absence seizures), 20 (17.86\%) seizures were generalized onset tonic-clonic seizures (GTCs), and 4 (3.57\%) seizures were non-convulsive seizures. Mean seizure severity, as measured by National Hospital Seizure Severity Scale, was $13.938(4-22)$.

Ninety-six $(85.71 \%)$ seizures were recorded during non-rapid eye movement (NREM) stage of sleep, 16 (14.29\%) seizures during awake state, and no seizures were recorded during REM stage of sleep. Thirty nine (34.82\%) seizures had generalized onset by EEG and 73 (65.18\%) seizures had focal onset by EEG. The ictal onset zone was frontal in 36 (32.14\%) seizures, temporal in $31(27.68 \%)$ seizures, parietal in $6(5.36 \%)$ seizures, and generalized in 39 (34.82\%) seizures. Thirty-two (28.57\%) seizures were lateralized to the left side, 41 (36.61\%) seizures to the right side, and 39 (34.82\%) seizures were generalized. While investigating ictal EEG patterns between participants, rhythmic spike and wave discharge were evident in 39 (39.82\%) seizures; paroxysmal fast activity in 55 (49.11\%) seizures; rhythmical evolving theta, delta, and alpha frequencies in 14 (12.5\%) seizures; and rhythmic polyspike and wave in 4 (3.57\%) seizures.

ICA was recorded in 36 (32.14\%) seizures with mean duration of 31.389 (20-55) s. Oxygen desaturation was mild in $25(22.32 \%)$ seizures and moderate in 8 (7.14\%) seizures. There was no change in heart rate in 77 $(68.75 \%)$ seizures, yet there was tachycardia in 32 (28.57\%) seizures and bradycardia in $3(2.68 \%)$ seizures.

While investigating correlations between incidence of ICA and different parameters, there were no statistically significant correlations with age, gender of patients, age of onset, duration of illness, frequency of seizures, and seizure severity. Also there were no statistically significant correlations with MRI brain finding, past history of status epilepticus occurrence, response to first antiseizure medication, drug resistance, and type or number of antiseizure medications used. However, there were statistically significant correlations with family history of epilepsy ( $p$ value $<0.037$ ), type of seizure as FOIA motor onset with automatism was evident in $44.44 \%$ of ICA events ( $p$ value $<0.001$ ), ictal onset zone as temporal lobe epilepsy was associated with ICA presence in comparison to extratemporal epilepsy $77.78 \%$ ( $p$ value $<0.001$ ), and seizure lateralization to the left hemisphere was associated with higher incidence of ICA ( $p$ value $<0.001)$ (Table 1). Also there were statistically significant correlations with state of the patient at seizure onset as all ICA events were recorded during non-REM stage of sleep ( $p$ value $<0.003)$ and ictal pattern as paroxysmal fast activity occurred in $69.44 \%$ of ICA events ( $p$ value $<0.018$ ). ICA was the only semiological feature in 4 (11.11\%) seizures (Table 2). On the other hand, duration of ICA showed statistically significant correlation only with severity of seizure ( $p$ value $<0.028$ ).

While investigating correlations between incidence of oxygen desaturation and different parameters, there was a statistically significant correlation with type of seizure (FOIA motor onset with automatism). It was associated with mild desaturation in 12 events and moderate desaturation in 4 events ( $p$ value $<0.001$ ). Temporal ictal onset zone was associated with mild desaturation in 17 events and moderate desaturation in 8 events ( $p$ value $<$ 0.001). Seizure lateralization to the left hemisphere was associated with higher incidence of oxygen desaturation ( $p$ value $<0.001$ ) (Table 3 ). Also there were statistically significant correlations with state of the patient at seizure onset as all oxygen desaturation events were recorded during non-REM stage of sleep ( $p$ value $<0.002$ ) and ictal pattern as paroxysmal fast activity was associated with mild desaturation in 19 events and moderate desaturation in 6 events ( $p$ value $<0.007)$ (Table 4).

While investigating correlations between incidence of heart rate changes and different parameters, there were statistically significant correlations with type of seizure as FOIA motor onset with automatism was associated with tachycardia in 9 events and bradycardia in 3 events ( $p$ value $<0.001$ ), temporal ictal onset zone was associated with tachycardia in 24 events and bradycardia in 3 events ( $p$ value $<0.001)$, and seizure lateralization to the left hemisphere was associated with higher incidence of tachycardia while the recorded 3 events of bradycardia was associated with lateralization to the right side ( $p$ value $<0.001)$ (Table 5). Also there were statistically significant correlations with state of the patient at seizure onset as all events of heart rate changes were recorded during non-REM stage of sleep ( $p$ value $<0.065$ ) and ictal EEG pattern as paroxysmal fast activity was associated with tachycardia in 23 events and bradycardia in 3 events $(p$ value $<0.016)$ (Table 6). Seizure severity had statistically significant correlation with incidence of tachycardia ( $p$ value $<0.001)$.

\section{Discussion}

This study suggests that ICA is a semiological feature exclusive to focal epilepsy and can be the only clinical manifestation of focal seizures as postulated in other studies [5]. Temporal lobe epilepsy and frontal lobe 
Table 1 Correlations between ICA occurrence and seizure semiology, lateralization, and ictal onset zone

\begin{tabular}{|c|c|c|c|c|}
\hline & \multicolumn{2}{|c|}{$\begin{array}{l}\text { ICA } \\
\text { Yes }\end{array}$} & \multicolumn{2}{|c|}{ Chi-square } \\
\hline & $N$ & $\%$ & $\overline{x^{2}}$ & $P$ value \\
\hline \multicolumn{5}{|l|}{ Type of seizure semiology } \\
\hline Yes & 4 & 11.11 & 46.793 & $<0.001^{*}$ \\
\hline FOIA motor onset with automatism & 16 & 44.44 & & \\
\hline FOIA nonmotor onset & 5 & 13.89 & & \\
\hline FOIA motor onset hyperkinetic or FOA motor onset tonic or clonic & 4 & 11.11 & & \\
\hline FBTCS & 7 & 19.44 & & \\
\hline Absence & 0 & 0.00 & & \\
\hline GTCs & 0 & 0.00 & & \\
\hline Non convulsive seizure & 4 & 11.11 & & \\
\hline \multicolumn{5}{|l|}{ Ictal onset zone } \\
\hline Frontal & 6 & 16.67 & 70.540 & $<0.001^{*}$ \\
\hline Temporal & 28 & 77.78 & & \\
\hline Parietal & 2 & 5.56 & & \\
\hline Generalized & 0 & 0.00 & & \\
\hline \multicolumn{5}{|l|}{ Seizure lateralization } \\
\hline Left & 20 & 55.56 & 32.884 & $<0.001^{*}$ \\
\hline Right & 16 & 44.44 & & \\
\hline Generalized & 0 & 0.00 & & \\
\hline
\end{tabular}

FBTCS focal onset to bilateral tonic-clonic seizures, FOA focal onset aware, FOIA focal onset with impaired awareness, GTCs generalized onset tonic-clonic seizures, $X^{2}$ chi-square value

*Statistically significant

epilepsy accounted for majority of focal epilepsies associated with apnea, yet temporal lobe epilepsy had a greater association with apnea than frontal lobe epilepsy. FOIA motor onset with automatisms semiology, typical of temporal lobe epilepsy, was much more likely to produce ICA. So presence of ICA may help determine focal epilepsies and they may also suggest localization of

Table 2 Correlations between ICA occurrence and ictal EEG pattern and state at seizure onset

\begin{tabular}{|c|c|c|c|c|}
\hline & \multicolumn{2}{|c|}{$\begin{array}{l}\text { ICA } \\
\text { Yes }\end{array}$} & \multicolumn{2}{|c|}{ Chi-square } \\
\hline & $\bar{N}$ & $\%$ & $\overline{x^{2}}$ & $P$ value \\
\hline \multicolumn{5}{|l|}{ State at seizure onset } \\
\hline Awake & 0 & 0.00 & 8.842 & $0.003^{*}$ \\
\hline Non REM & 36 & 100.00 & & \\
\hline \multicolumn{5}{|l|}{ Ictal EEG pattern } \\
\hline Rhythmic spike and wave & 7 & 19.44 & 10.047 & $0.018^{*}$ \\
\hline Paroxysmal fast activity & 25 & 69.44 & & \\
\hline Rhythmical theta, delta, alpha & 4 & 11.11 & & \\
\hline Rhythmic polyspike and wave & 0 & 0.00 & & \\
\hline
\end{tabular}

EEG electroencephalography, REM rapid eye movement, $x^{2}$ chi-square value *Statistically significant seizures to the temporal lobe. Temporal lobe as a cause of ICA was previously postulated with direct electrical cortical stimulation studies in humans that direct to highly reproducible apnea responses with low intensity $[12,13]$, unilateral (left or right) amygdalar and hippocampal stimulation [14-16].

ICA showed higher incidence with higher seizure severity as measured by National Hospital Seizure Severity Scale. As far as our knowledge, this point was not postulated previously.

Seizure lateralization to the left hemisphere was found to have greater incidence of ICA. That was supported by previous studies $[5,17]$. On the other stand, another study showed that seizure lateralization to the right hemisphere was found to have greater incidence of ICA [18].

Mean and range ICA durations in this study were 31 and $20-55 \mathrm{~s}$, respectively, suggesting that in most cases ICA is self-limiting and unlikely to be a SUDEP concern. However, prolonged ICA ( $\geq 60$ s) was not recorded within our study, yet it was recorded in previous studies, was associated with severe hypoxemia, and was thought to be a predictor of SUDEP occurrence [5].

The low incidence of ictal bradycardia with ICA in this study was reported in a minority of seizures in one previous series [19] which is surprising because bradycardia is 
Table 3 Correlations between oxygen desaturation and seizure semiology, lateralization, and ictal onset zone

\begin{tabular}{|c|c|c|c|c|c|c|}
\hline & \multicolumn{4}{|c|}{ Oxygen desaturation } & \multirow{2}{*}{\multicolumn{2}{|c|}{ Chi-square }} \\
\hline & \multicolumn{2}{|c|}{ Mild } & \multicolumn{2}{|c|}{ Moderate } & & \\
\hline & $N$ & $\%$ & $N$ & $\%$ & $x^{2}$ & $P$ value \\
\hline \multicolumn{7}{|l|}{ Type of seizure semiology } \\
\hline FOIA motor onset with automatism & 12 & 48.00 & 4 & 50.00 & 41.825 & $<0.001^{*}$ \\
\hline FOIA nonmotor onset & 4 & 16.00 & 1 & 12.50 & & \\
\hline FOIA motor onset hyperkinetic or FOA motor onset tonic or clonic & 2 & 8.00 & 2 & 25.00 & & \\
\hline FBTCS & 6 & 24.00 & 1 & 12.50 & & \\
\hline Absence & 0 & 0.00 & 0 & 0.00 & & \\
\hline GTCs & 0 & 0.00 & 0 & 0.00 & & \\
\hline Non convulsive seizure & 1 & 4.00 & 0 & 0.00 & & \\
\hline \multicolumn{7}{|l|}{ Ictal onset zone } \\
\hline Frontal & 6 & 24.00 & 0 & 0.00 & 62.296 & $<0.001^{*}$ \\
\hline Temporal & 17 & 68.00 & 8 & 100.00 & & \\
\hline Parietal & 2 & 8.00 & 0 & 0.00 & & \\
\hline Generalized & 0 & 0.00 & 0 & 0.00 & & \\
\hline \multicolumn{7}{|l|}{ Seizure lateralization } \\
\hline Left & 14 & 56.00 & 3 & 37.50 & 28.007 & $<0.001^{*}$ \\
\hline Right & 11 & 44.00 & 5 & 62.50 & & \\
\hline Generalized & 0 & 0.00 & 0 & 0.00 & & \\
\hline
\end{tabular}

FBTCS focal onset to bilateral tonic-clonic seizures, FOA focal onset aware, FOIA focal onset with impaired awareness, GTCs generalized onset tonic-clonic seizures, $x^{2}$ chi-square value

*Statistically significant

considered as a normal response to hypoventilation. Ictal tachycardia is common [20] and may have overcome any physiological tendency to bradycardia in these patients. Therefore, the combination of ICA and bradycardia appears rare, but when it occurs, it may indicate a potentially deleterious, high vagal tone phenotype in seizure patients in the SUDEP context. An Egyptian case presentation recorded ictal bradycardia and asystole in a patient with temporal lobe epilepsy [21]. The frequently observed tachycardia apnea combination in this study may reflect a self-limiting seizure manifestation and that was supported by multiple previous studies [7].

Seizure lateralization to the left hemisphere was found to have greater incidence of ictal tachycardia. And the three recorded events of ictal bradycardia were associated with seizure lateralization to the right hemisphere.

Table 4 Correlations between oxygen desaturation and ictal EEG pattern and state at seizure onset

\begin{tabular}{|c|c|c|c|c|c|c|}
\hline & \multicolumn{4}{|c|}{ Oxygen desaturation } & \multirow{2}{*}{\multicolumn{2}{|c|}{ Chi-square }} \\
\hline & \multicolumn{2}{|c|}{ Mild } & \multicolumn{2}{|c|}{ Moderate } & & \\
\hline & $\bar{N}$ & $\%$ & $\bar{N}$ & $\%$ & $\overline{x^{2}}$ & $P$ value \\
\hline \multicolumn{7}{|l|}{ State at seizure onset } \\
\hline Awake & 0 & 0.00 & 0 & 0.00 & 7.797 & $0.020^{*}$ \\
\hline Non REM & 25 & 100.00 & 8 & 100.00 & & \\
\hline \multicolumn{7}{|l|}{ Ictal EEG pattern } \\
\hline Rhythmic spike and wave & 6 & 24.00 & 0 & 0.00 & 17.744 & $0.007^{*}$ \\
\hline Paroxysmal fast activity & 19 & 76.00 & 6 & 75.00 & & \\
\hline Rhythmical evolving theta, delta, alpha & 0 & 0.00 & 2 & 25.00 & & \\
\hline Rhythmic polyspike and wave & 0 & 0.00 & 0 & 0.00 & & \\
\hline
\end{tabular}

EEG electroencephalography, REM rapid eye movement, $x^{2}$ chi-square value *Statistically significant 
Table 5 Correlations between heart rate changes and seizure semiology, lateralization, and ictal onset zone

\begin{tabular}{|c|c|c|c|c|c|c|}
\hline & \multicolumn{4}{|c|}{ Arrhythmia } & \multirow{2}{*}{\multicolumn{2}{|c|}{ Chi-Square }} \\
\hline & \multicolumn{2}{|c|}{ Tachycardia } & \multicolumn{2}{|c|}{ Bradycardia } & & \\
\hline & $\bar{N}$ & $\%$ & $\bar{N}$ & $\%$ & $x^{2}$ & $P$ value \\
\hline \multicolumn{7}{|l|}{ Type of seizure semiology } \\
\hline FOIA motor onset with automatism & 9 & 28.13 & 3 & 100.00 & 45.688 & $<0.001^{*}$ \\
\hline FOIA nonmotor onset & 6 & 18.75 & 0 & 0.00 & & \\
\hline FOIA motor onset hyperkinetic or FOA motor onset tonic or cloni & 4 & 12.50 & 0 & 0.00 & & \\
\hline FBTCS & 7 & 21.88 & 0 & 0.00 & & \\
\hline Absence & 0 & 0.00 & 0 & 0.00 & & \\
\hline GTCs & 2 & 6.25 & 0 & 0.00 & & \\
\hline Non convulsive seizure & 4 & 12.50 & 0 & 0.00 & & \\
\hline \multicolumn{7}{|l|}{ Ictal onset zone } \\
\hline Frontal & 6 & 18.75 & 0 & 0.00 & 64.630 & $<0.001^{*}$ \\
\hline Temporal & 24 & 75.00 & 3 & 100.00 & & \\
\hline Parietal & 0 & 0.00 & 0 & 0.00 & & \\
\hline Generalized & 2 & 6.25 & 0 & 0.00 & & \\
\hline \multicolumn{7}{|l|}{ Seizure lateralization } \\
\hline Left & 17 & 53.13 & 0 & 0.00 & 26.049 & $<0.001^{*}$ \\
\hline Right & 13 & 40.63 & 3 & 100.00 & & \\
\hline Generalized & 2 & 6.25 & 0 & 0.00 & & \\
\hline
\end{tabular}

FBTCS focal onset to bilateral tonic-clonic seizures, FOA focal onset aware, FOIA focal onset with impaired awareness, GTCs generalized onset tonic-clonic seizures, $x^{2}$ chi-square value

*Statistically significant

The correlation between seizure onset lateralization and ictal tachycardia was inconsistent across most studies [7]. In a study using invasive electrodes, no difference in ictal heart rate increase could be found in seizures arising from the left or right temporal lobe [22].
On the other hand, other studies showed that while stimulation of the left-sided insula in humans causes bradycardia, right-sided stimulation results in tachycardia [23], suggesting that right-sided seizures would be more prone to result in ictal tachycardia. Indeed, some

Table 6 Correlations between heart rate changes and ictal EEG pattern and state at seizure onset

\begin{tabular}{|c|c|c|c|c|c|c|}
\hline & \multicolumn{4}{|c|}{ Arrhythmia } & \multirow{2}{*}{\multicolumn{2}{|c|}{ Chi-square }} \\
\hline & \multicolumn{2}{|c|}{ Tachycardia } & \multicolumn{2}{|c|}{ Bradycardia } & & \\
\hline & $N$ & $\%$ & $N$ & $\%$ & $x^{2}$ & $P$ value \\
\hline \multicolumn{7}{|l|}{ State at seizure onset } \\
\hline Awake & 1 & 3.13 & 0 & 0.00 & 5.452 & 0.065 \\
\hline Non REM & 31 & 96.88 & 3 & 100.00 & & \\
\hline Right & 13 & 40.63 & 3 & 100.00 & & \\
\hline Generalized & 2 & 6.25 & 0 & 0.00 & & \\
\hline \multicolumn{7}{|l|}{ Ictal EEG pattern } \\
\hline Rhythmic spike and wave & 5 & 15.63 & 0 & 0.00 & 15.580 & $0.016^{*}$ \\
\hline Paroxysmal fast activity & 23 & 71.88 & 3 & 100.00 & & \\
\hline Rhythmical evolving theta, delta, alpha & 4 & 12.50 & 0 & 0.00 & & \\
\hline Rhythmic polyspike and wave & 0 & 0.00 & 0 & 0.00 & & \\
\hline
\end{tabular}

EEG electroencephalography, $R E M$ rapid eye movement, $x^{2}$ chi-square value 
studies have suggested a more pronounced increase in heart rate during seizures arising from the right hemisphere compared to the left one [24].

We should consider some limitations in this study. Our results were based on a relatively few seizures in the primary generalized epilepsy group. Loss of plethysmographic breathing signal due to movement artifact resulted in difficult comment on ICA. The exclusion of events that were contaminated by artifact may also underestimate incidence of ICA in extratemporal seizures, as these may be more likely to induce vigorous movements (for example, hypermotor movements) than temporal seizures.

\section{Conclusion}

The main findings of this study were that ICA occurred exclusively in focal seizures and tachycardia percentage was more with focal seizures. And both had higher magnitude in temporal lobe epilepsy in comparison to other types and in seizures with left hemispheric onset. Yet larger sample should be studied including focal and generalized epilepsy.

\section{Abbreviations \\ AVM: Arteriovenous malformation; DRE: Drug-resistant epilepsy; ECG: Electrocardiography; EEG: Electroencephalography; FBTCS: Focal onset to bilateral tonic-clonic seizures; FOA: Focal onset aware; FOIA: Focal onset with impaired awareness; GTCs: Generalized onset tonic-clonic seizures; ICA: Ictal central apnea; IQR: Interquartile range; ILAE: International League Against Epilepsy; MORTEMUS: MORTality in Epilepsy Monitoring Unit Study; MRI: Magnetic resonance imaging; NREM: Non-rapid eye movement; PNES: Psychogenic nonepileptic seizures; REM: Rapid eye movement; SD: Standard deviation; SPSS: Statistical package for Social Science; SUDEP: Sudden unexpected death in epilepsy; VEEG: Video- electroencephalography}

\section{Acknowledgements}

Not applicable.

\section{Authors' contributions}

$M O, A G, N E$, and YA conceived of the study and participated in its design and coordination and helped to draft the manuscript. RY and MW participated in the design of the study and performed the statistical analysis. All authors have agreed to conditions noted on the Authorship Agreement Form and have read and approved the final version submitted.

\section{Funding}

Self-financing

\section{Availability of data and materials}

Dataset is available as master sheet in Excel format and publicly available in Neurology Department, Ain Shams University, through communicating with the corresponding author.

\section{Declarations}

\section{Ethics approval and consent to participate}

The study protocol was approved by Ain Shams University, Faculty of Medicine Research Ethical Committee FWA 000017585 in 2018. Written informed consent was obtained from the patients participating in the study, or their first-degree relatives if the patient was unable to provide consent, after informing them about the study rationale and their right to withdraw from the study at any time without any consequences.
Consent for publication

Not applicable.

\section{Competing interests}

All authors declare no competing interests.

Received: 21 December 2020 Accepted: 1 March 2021

Published online: 16 March 2021

\section{References}

1. Lamberts RJ, Thijs RD, Laffan A, Langan Y, Sander JW. Sudden unexpected death in epilepsy: people with nocturnal seizures may be at highest risk. Epilepsia. 2012;53(2):253-7.

2. Devinsky O, Hesdorffer DC, Thurman DJ, Lhatoo S, Richerson G. Sudden unexpected death in epilepsy: epidemiology, mechanisms, and prevention. Lancet Neurol. 2016;15(10):1075-88.

3. Sveinsson O, Andersson T, Carlsson S, Tomson T. The incidence of SUDEP: a nationwide population-based cohort study. Neurology. 2017:89(2):170-7.

4. Nascimento FA, Tseng ZH, Palmiere C, Maleszewski JJ, Shiomi T, McCrillis A, et al. Pulmonary and cardiac pathology in sudden unexpected death in epilepsy (SUDEP). Epilepsy Behav. 2017;73:119-25.

5. Lacuey N, Zonjy B, Hampson JP, Rani MRS, Zaremba A, Sainju RK, et al. The incidence and significance of periictal apnea in epileptic seizures. Epilepsia. 2018;59(3):573-82

6. Vilella L, Lacuey N, Hampson JP, Rani MR, Sainju RK, Friedman D, et al. Postconvulsive central apnea as a biomarker for sudden unexpected death in epilepsy (SUDEP). Neurology. 2018. https://doi.org/10.1212/WNL. 0000000000006785

7. Eggleston KS, Olin BD, Fisher RS. Ictal tachycardia: the head-heart connection. Seizure. 2014;23:496-505.

8. Stöllberger C, Finsterer J. Cardiorespiratory findings in sudden unexplained/ unexpected death in epilepsy (SUDEP). Epilepsy Res. 2004;59:51-60.

9. Kwan P, Arzimanoglou A, Berg A, Brodie M, Allen Hauser W, Mathern G, French J, et al. Definition of drug resistant epilepsy: consensus proposal by the ad hoc Task Force of the ILAE Commission on Therapeutic Strategies. Epilepsia. 2009;51(6):1069-77.

10. O'Donoghue MF, Duncan JS, Sander JW. The National Hospital Seizure Severity Scale: a further development of the Chalfont Seizure Severity Scale. Epilepsia. 1996;37(6):563-71. https://doi.org/10.1111/j.1528-1157.1996.tb0061 0.x. PMID: 8641234

11. Fisher $R$. The new classification of seizures by the International League Against Epilepsy 2017. Curr Neurol Neurosci Rep. 2017;17:48.

12. Pool $J$, Ransohoff $J$. Autonomic effects on stimulating rostral portion of cingulate gyri in man. J Neurophysiol. 1949;12:385-92.

13. Chapman WP, Livingston RB, Livingston KE. Frontal lobotomy and electrical stimulation of orbital surface of frontal lobes; effect on respiration and on blood pressure in man. Arch Neurol Psychiatry. 1949;62:701-16.

14. Lacuey N, Zonjy B, Londono L, Samden DL. Amygdala and hippocampus are symptomatogenic zones for central apneic seizures. Neurology. 2017;88: 701-5.

15. Dlouhy BJ, Gehlbach BK, Kreple CJ, Kawasaki H, Oya H, Buzza C, Granner MA et al. Breathing inhibited when seizures spread to the amygdala and upon amygdala stimulation. J Neurosci. 2015;35(28):10281-9.

16. Kaada BR, Jasper $\mathrm{H}$. Respiratory responses to stimulation of temporal pole, insula, and hippocampal and limbic gyri in man. AMA Arch Neurol Psychiatry. 1952;68:609-19.

17. Vilella L, Lacuey N, Hampson JP, Rani MRS, Loparo K, Sainju RK, Allen L. Recurrence, and risk factors for peri-ictal central apnea and sudden unexpected death in epilepsy incidence. Front Neurol. 2019:10:166.

18. Tio E, George W, Elizabeth M, Stephan S. Ictal central apneas in temporal lobe epilepsies. Epilepsy Behav. 2020;112:107434. https://doi.org/10.1016/j. yebeh.2020.107434. Epub 2020 Sep 8. PMID: 32916581; PMCID: PMC7658036

19. Nashef L, Walker F, Allen P, Sander JW, Shorvon SD, Fish DR. Apnoea and bradycardia during epileptic seizures: relation to sudden death in epilepsy. J Neurol Neurosurg Psychiatry. 1996;60(3):297-300.

20. Chen W, Guo CL, Zhang PS, Chong L, Hui Q, Jian-Guo Z. Heart rate changes in partial seizures: analysis of influencing factors among refractory patients. BMC Neurol. 2014;14:135.

21. Kishk N, Nawito A, El-Damaty A, Ragab A. Ictal asystole: a case presentation. BMC Neurol. 2018;18(1):100. 
22. Saleh Y, Kirchner A, Pauli E, Hilz MJ, Neundorfer B, Stefan H. Temporal lobe epilepsies: influence of the focus side on the autonomic heart rate? Nervenarzt. 2020;71:477-80.

23. Oppenheimer SM, Gelb A, Girvin JP, Hachinski VC. Cardiovascular effects of human insular cortex stimulation. Neurology. 1992;42(9):1727.

24. Shimmura M, Uehara T, Ogata K, Shigeto H, Maeda T, Sakata A, et al. Higher postictal parasympathetic activity following greater ictal heart rate increase in right- than left-sided seizures. Epilepsy Behav. 2019;97:161-8.

\section{Publisher's Note}

Springer Nature remains neutral with regard to jurisdictional claims in published maps and institutional affiliations.

\section{Submit your manuscript to a SpringerOpen ${ }^{\odot}$ journal and benefit from:}

- Convenient online submission

- Rigorous peer review

- Open access: articles freely available online

High visibility within the field

- Retaining the copyright to your article

Submit your next manuscript at $\boldsymbol{\nabla}$ springeropen.com 